Published in final edited form as:

Autism Res. 2020 January ; 13(1): 93-103. doi:10.1002/aur.2220.

\title{
Discrepancies Between Parent and Child Ratings of Anxiety in Children With Autism Spectrum Disorder
}

\author{
Carla B. Kalvin, Carolyn L. Marsh, Karim Ibrahim, Theresa R. Gladstone, Diana Woodward, \\ Heidi Grantz, Pamela Ventola, Denis G. Sukhodolsky \\ Child Study Center, Yale University School of Medicine, New Haven, Connecticut
}

\section{Abstract}

Co-occurring anxiety is common in children with autism spectrum disorder (ASD). However, inconsistencies across parent and child reports of anxiety may complicate the assessment of anxiety in this population. The present study examined parent and child anxiety ratings in children with ASD with and without anxiety disorders and tested the association between parent-child anxiety rating discrepancy and ASD symptom severity. Participants included children aged 8-16 years in three diagnostic groups: ASD with co-occurring anxiety disorders (ASD + Anxiety; $n=$ 34), ASD without co-occurring anxiety disorders (ASD; $n=18$ ), and typically developing healthy controls (TD; $n=50$ ). Parents and children completed ratings of child anxiety using the Multidimensional Anxiety Rating Scale. Patterns of parent and child anxiety ratings differed among the three groups, with parent ratings exceeding child ratings only in the ASD + Anxiety group. Parents reported higher levels of child anxiety in the ASD + Anxiety versus ASD group, whereas children reported comparable levels of anxiety in the two groups. Among children with ASD, ASD symptom severity was positively associated with the degree to which parent ratings exceeded child ratings. Results suggest that children with ASD and co-occurring anxiety disorders endorse some anxiety symptoms but may underreport overall levels of anxiety. In addition, ASD symptom severity might increase discrepancies in parent-child anxiety ratings. These findings suggest a unique and valuable role of child anxiety ratings and suggest that both parent and child anxiety ratings should be considered in light of children's ASD symptom severity and used to guide further assessment.

\section{Lay Summary}

Children with autism spectrum disorder (ASD) commonly experience anxiety; yet, their perceptions of their anxiety might differ from their parents' perceptions. This study found that, while children with ASD and anxiety disorders acknowledge some anxiety, their parents report them as having higher levels of anxiety. Also, child and parent perceptions of anxiety may differ

\footnotetext{
Address for correspondence and reprints: Carla B. Kalvin, Child Study Center, Yale University School of Medicine, 230 S. Frontage Road, New Haven, CT 06520. carla.kalvin@yale.edu.

Conflict of Interest

Dr. Sukhodolsky receives royalties from Guilford Press for a treatment manual on CBT for anger and aggression in children. Other authors (C.B.K., C.L.M., K.I., T.R.G., D.W., H.G., and P.V.) have no biomedical financial interests or potential conflicts of interest to declare related to this present study.

Supporting Information

Additional supporting information may be found online in the Supporting Information section at the end of the article.
} 
more for children with more severe ASD symptoms. How these findings may guide research and clinical practice is discussed.

\section{Keywords}

anxiety; assessment; autism spectrum disorder; comorbidity; self-report

\section{Introduction}

Co-occurring anxiety is common among children with autism spectrum disorder (ASD), with approximately $40 \%$ of children with ASD meeting criteria for at least one anxiety disorder [Lecavalier et al. 2019]. Comorbid anxiety disorders can compromise daily functioning and exacerbate core symptoms of ASD, including impairments in social communication [Duvekot, van der Ende, Verhulst, \& Greaves-Lord 2018]. Over the past 10 years, considerable progress has been made in the assessment and treatment of anxiety in children with ASD [Scahill et al. 2019; Sukhodolsky, Bloch, Panza, \& Reichow 2013]. However, relatively little research has addressed the issue of discrepancies in parent and child ratings of anxiety in ASD.

Collecting information from multiple informants, particularly parents and children, is considered best practice in the assessment of childhood psychological disorders [Hunsley \& Mash 2007]. However, patterns of inconsistent ratings of symptomatology across parents and children occur frequently, with correlations between parent and child ratings ranging from 0.2 to 0.3 and reflecting lower levels of agreement for internalizing $(r=0.26)$ versus externalizing ( $r=0.32)$ symptoms [Achenbach 2006; De Los Reyes et al. 2015]. Inconsistent ratings result from multiple factors, such as differences in informant perspectives and attributions, and are particularly common in the assessment of anxiety [De Los Reyes \& Kazdin 2005; Foley et al. 2005; Miller, Martinez, Shumka, \& Baker 2014; Silverman \& Ollendick 2005].

Collecting information about anxiety from both parents and children and reconciling potential discrepancies in ratings may be particularly important in ASD, as subjective distress can be difficult to infer from observable behavior or dissociate from the core symptoms of ASD. For example, parents of children with ASD may have difficulty differentiating core symptoms of the disorder (e.g., repetitive behavior, rigidity) from anxiety symptoms [Storch et al. 2012]. At the same time, children with ASD may struggle to accurately report on their emotions [Wood \& Gadow 2010] due to limitations in emotional awareness [Bal et al. 2010; Losh \& Capps 2006] and deficits in communication. Together, these patterns may contribute to discrepancies between parent and child reports of anxiety and complicate the assessment process. As such, a better understanding of parent-child discrepancies in anxiety reporting in this population is warranted.

Research on parent-child agreement in anxiety ratings in children with ASD has yielded mixed findings. Six recent studies exploring anxiety in children with ASD demonstrated fair to moderate agreement across parent and child anxiety ratings, with correlations ranging from $r=0.36$ to 0.70 [Blakeley-Smith, Reaven, Ridge, \& Hepburn 2012; Farrugia \& Hudson 
2006; Magiati, Chan, Tan, \& Poon 2014; Ooi et al. 2016; Ozsivadjian, Hibberd, \& Hollocks 2014; White, Schry, \& Maddox 2012]. In contrast, five separate studies investigating anxiety ratings in children with ASD found poor agreement between parent and child raters, evidenced by correlations ranging from $r=0.13$ to 0.25 or lack of agreement when considering diagnostic thresholds [Greenaway \& Howlin 2010; Lopata et al. 2010; May, Cornish, \& Rinehart 2015; Mazefsky, Kao, \& Oswald 2011; Storch et al. 2012].

Additionally, several studies of children with ASD have demonstrated higher parent versus child ratings of anxiety [Bitsika \& Sharpley 2015; Blakeley-Smith et al. 2012; Lopata et al. 2010; Ozsivadjian et al. 2014; Russell \& Sofronoff 2005; White et al. 2012], whereas other studies have reported the opposite pattern [Hurtig et al. 2009; Magiati et al. 2014; Ooi et al. 2016]. One recent study reported no difference in parent and child anxiety ratings in this population [Burrows et al. 2018]. The lack of consistent findings on agreement between parent and child anxiety ratings in children with ASD underscores the need to better understand factors contributing to discrepancies.

Sample characteristics such as age, IQ, and presence of a co-occurring anxiety disorder may contribute to discrepancies in parent and child anxiety ratings. In particular, some research suggests that a pattern of heightened parent versus child ratings may be particularly pronounced for older children with ASD [Bitsika \& Sharpley 2015] and that, in children with ASD with IQ scores above 75, parent-child anxiety rating discrepancies may be attenuated for children with higher IQ [Blakeley-Smith et al. 2012; Ooi et al. 2016]. With regard to presence of co-occurring anxiety, only three studies have utilized samples of children with ASD and confirmed comorbid anxiety disorder diagnoses [Mazefsky et al. 2011; Storch et al. 2012; White et al. 2012], while other studies have included samples of children with ASD without reporting on presence or absence of co-occurring psychiatric disorders. However, presence of a co-occurring anxiety disorder may be an important factor in agreement versus disagreement in parent and child anxiety ratings. For example, clinically significant anxiety may be more readily observable to parents, leading to increased parent ratings. And if children with ASD underreport their anxiety symptoms due to difficulties with self-awareness or communication that are conferred by the core symptoms of ASD, there could be increased discrepancy in parent-child anxiety ratings. Indeed, in the studies of children with ASD and comorbid anxiety disorders comparing parent and child anxiety ratings, parents reported more anxiety than children [Storch et al. 2012; White et al. 2012]. Yet, no studies have examined parent and child anxiety ratings in children with ASD who have a co-occurring anxiety disorder compared to children with ASD without anxiety. In the present study, we address this gap in the literature by including two groups of children with ASD, one with comorbid anxiety diagnoses and one without comorbid anxiety diagnoses.

Children's ASD symptom severity may also impact parent and child anxiety ratings and influence parent-child rating discrepancy. Specifically, symptoms related to social communication and interaction, such as deficits in social-emotional reciprocity and awareness of internal emotional experiences, may result in reduced child ratings of anxiety. Additionally, parents may conflate ASD and anxiety symptoms, leading parents to rate higher levels of anxiety in children with more severe ASD symptomatology. Supporting this pattern, in a sample of 30 boys with ASD, greater ASD severity was associated with higher parent ratings of anxiety [Ozsivadjian et al. 2014]. Furthermore, in two studies examining 
ASD symptom severity and anxiety rating agreement, greater ASD symptom severity was associated with less agreement between parent and child anxiety ratings [Kaat \& Lecavalier 2015; Magiati et al. 2014].

In this study, we examined parent and child anxiety ratings in well-characterized samples of verbally fluent children with ASD with and without comorbid anxiety disorders. We also included a typically developing control group to allow for comparisons to children without ASD. Our first aim was to compare parent and child anxiety ratings in three groups of children: ASD and comorbid anxiety (ASD + Anxiety), ASD without comorbid anxiety (ASD), and typically developing (TD) children without any psychiatric disorder. We predicted that parent and child anxiety ratings would be highest in the ASD + Anxiety group and lowest in the TD group. We also predicted that parent ratings would be higher than child ratings in the ASD + Anxiety group and that child ratings would be higher than parent ratings in the TD group. We had no a priori hypotheses regarding relative levels of parent and child ratings in the ASD group. Our second aim was to test the association of parentchild anxiety rating discrepancy with ASD symptom severity, as well as with age, gender, and IQ. We predicted that ASD symptom severity would be positively associated with parent-child anxiety rating discrepancy, marked by higher parent versus child anxiety ratings.

\section{Methods}

\section{Participants}

Participants included 102 children (aged 8-16 years) comprised of three groups: 34 children with ASD and co-occurring anxiety disorders (ASD + Anxiety group), 18 children with ASD without co-occurring anxiety disorders (ASD group), and 50 TD healthy controls (TD group). Participant demographics and clinical characteristics are shown in Table 1.

\section{Procedures}

ASD diagnosis was based on the Autism Diagnostic Interview-Revised (ADI-R) [Le Couteur, Lord, \& Rutter 2003] and the Autism Diagnostic Observation Schedule, 2nd edition (ADOS-2) [Lord et al. 2012], which were administered by a research-reliable clinician. For children with ASD, the Anxiety Disorders Interview Schedule for Children and Parents (ADIS-C/P) [Silverman \& Albano 1996] or the Schedule for Affective Disorders and Schizophrenia for School-Age Children-Present and Lifetime Version (K-SADS-PL) [Kaufman et al. 2016] was administered to assess for DSM-5 disorders, including anxiety disorders. The ADIS and K-SADS were administered by two licensed clinicians who were highly experienced in conducting structured diagnostic interviews in research studies of children with neurodevelopmental disorders including ASD. Each clinician received detailed instructions on administration of the ADIS and K-SADS and achieved 100\% agreement on three practice cases with a third clinician who was an expert in anxiety in ASD. Both the ADIS and K-SADS were conducted with parent and child, and when there were differences between parents and children, the raters used their clinical judgment to elicit information necessary to confirm each diagnosis. Inter-rater reliability of the ADIS and K-SADS in this study was assessed by Cohen's kappa in a subset of 10 ADIS and 13 K-SADS interviews 
that were videotaped for quality assurance purposes and rated by a second rater [Bujang \& Baharum 2017]. The Cohen's kappa coefficients for the diagnoses assigned based on the ADIS ranged from 0.62 to 1.0 (Generalized Anxiety Disorder-1.0; Social Anxiety Disorder - 0.74; Specific Phobia— 0.78; Separation Anxiety Disorder-1.0; Obsessive Compulsive Disorder-0.62; Oppositional Defiant Disorder-0.74; ADHD—1.0; and Major Depressive Disorder-1.0). The Cohen's kappa coefficients for the K-SADS ranged from 0.63 to 1.0 (Generalized Anxiety Disorder-0.63; Social Anxiety Disorder-0.76; Specific Phobia0.81; Separation Anxiety Disorder-1.0; OCD—1.0; Oppositional Defiant Disorder-0.83; ADHD-0.76; and Major Depressive Disorder-0.63). By convention, Cohen's kappa values from 0.61 to 0.80 indicate substantial agreement, and from 0.81 to 1.00 indicate almost perfect or perfect agreement [Landis \& Koch 1977].

Following administration of the ADIS or K-SADS, all diagnoses were confirmed by best estimate consensus diagnoses [Leckman, Sholomskas, Thompson, Belanger, \& Weissman 1982], by which all available information was reviewed, and any diagnostic disagreements were discussed and resolved by consensus. To measure full-scale IQ, the Differential Ability Scales-II [Elliott 2007] or the Wechsler Abbreviated Scale of Intelligence [Wechsler 1999] was administered to all children. In addition to the clinical assessments, all parents provided demographic and medical history information.

All children were required to have a full-scale IQ score $\geq 70$. In addition to a confirmed diagnosis of ASD, children in the ASD + Anxiety group were required to meet DSM-5 criteria for at least one anxiety disorder, and children in the ASD group were required to have no past or current anxiety disorders. Children in the TD group were required to have no past or current psychiatric disorders.

Children with ASD were recruited from the Yale Child Study Center Autism Program and participated in studies of behavioral therapy. For children who participated in clinical trials, this study reports baseline data (i.e., data collected before children received study interventions). TD children were recruited from the local community. Parents provided written informed consent and children provided written assent. All study procedures were approved by the Yale University School of Medicine Institutional Review Board and complied with ethical standards of the American Psychological Association.

\section{Measures}

The Multidimensional Anxiety Scale for Children, 2nd edition (MASC-2) [March 2012] is a 50-item scale that assesses anxiety symptoms in children and adolescents. On this measure, parents and children completed the parent report and child self-report versions, respectively, which include identical items aside from different pronouns to fit the reporter. Items on the MASC-2 are rated on a 4-point scale ranging from 0 (never) to 3 (often), with higher scores reflecting greater anxiety symptomatology. The MASC-2 consists of 10 subscales and a Total Anxiety scale and yields $T$-scores based on child age and gender, with $T$-scores $\succ 65$ indicating elevated levels of anxiety. The MASC-2 has good internal consistency for children with ASD in both parent report $(a=0.92)$ and child self-report $(a=0.90)$ [Kaat $\&$ Lecavalier 2015]. Both the parent report and child self-report had good internal reliability in the current total sample ( $a=0.94$ and $a=0.93$, respectively), as well as within the ASD + 
Anxiety ( $a=.90$ and $a=.92$ ), ASD ( $a=0.80$ and $a=0.95$ ), and TD groups ( $a=0.82$ and $a=0.89)$. Parent- and child-rated Total Anxiety $T$-scores were used for the analysis in the current study in order to make comparisons between parent and child ratings based on population-based age and gender norms.

The ADI-R [Le Couteur et al. 2003] and the ADOS-2 [Lord et al. 2012] were also used as indexes of ASD symptom severity. The ADI-R is a clinician-administered semi-structured interview conducted with the caregiver to assist with diagnosis of ASD. The clinician scores items based on the caregiver's responses to questions regarding the child's current and lifetime functioning. The ADI-R comprehensive algorithm yields total scores in the following domains: Qualitative Abnormalities in Reciprocal Social Interaction, Qualitative Abnormalities in Communication, and Restricted, Repetitive, and Stereotyped Patterns of Behavior (RRB). Higher scores indicate greater severity of ASD-related symptoms and have been linked to higher parent ratings of child anxiety [Hallett et al. 2013; Sukhodolsky et al. 2008].

The ADOS-2 is a clinician-administered observational assessment conducted with the child to evaluate and diagnose ASD. The clinician evaluates the child's language and communication, reciprocal social interaction, and stereotyped behaviors and restricted interests. All ADOS-2 administrations in the present study were conducted with Module 3, intended for verbally fluent children. The ADOS-2 Module 3 algorithm yields scores in the areas of Social Affect (SA) and Restricted and Repetitive Behavior (RRB), as well as a Total Score, which can be used to establish classification of autism, autism spectrum, or nonspectrum. Higher scores indicate greater severity of ASD-related symptoms and have been associated with higher scores on child anxiety ratings, including the MASC-2 [Kaat \& Lecavalier 2015].

\section{Data Analyses}

First, a mixed-design analysis of variance (ANOVA) was used to test differences in anxiety ratings across raters (i.e., parent, child) and diagnostic groups (i.e., ASD + Anxiety, ASD, and TD). Planned post hoc tests were conducted using the Holm-Bonferroni adjustment to correct for multiple comparisons. To examine agreement in parent and child anxiety ratings in each of the diagnostic groups, bivariate correlations were conducted within group.

Next, we examined the association of the discrepancy between parent and child anxiety ratings with child's age, gender, IQ, and ASD symptom severity as measured by the ADOS-2 and ADI-R. These analyses were conducted in the combined ASD sample (ASD + Anxiety group plus ASD group, $n=52$ ). Discrepancy in anxiety ratings was calculated using the method proposed by De Los Reyes and Kazdin [2004], which produces a difference score that is equally correlated with each set of informant ratings. Specifically, parent and child anxiety ratings (MASC-2 Total Anxiety $T$-scores) were first converted into $Z$ scores, and then the child anxiety $z$ scores were subtracted from the parent anxiety z scores to create the parent-child discrepancy score. The sign was retained, consistent with prior work [De Los Reyes \& Kazdin 2006]. The score reflected the extent to which a parent's rating of his/her child's anxiety exceeded the child's rating, with positive values reflecting higher parent (versus child) ratings, and negative values reflecting higher child (versus parent) 
ratings. Bivariate correlations between the discrepancy score and age, gender, IQ, and the ASD symptom severity variables were conducted. Lastly, the variables significantly associated with the discrepancy score were entered into a hierarchical linear regression to test their relative and combined contributions to parent-child anxiety rating discrepancy. All analyses were conducted using SPSS v24 (IBM corp. 2017).

\section{Results}

Demographic and clinical characteristics of the three diagnostic groups are reported in Table 1. There were significant differences in IQ score, with higher scores in the TD versus ASD + Anxiety group, and in ethnicity, with a greater proportion of Hispanic/Latino children in the ASD + Anxiety versus ASD group. However, it is important note that there were no significant differences in IQ between the ASD subgroups. There were also no significant differences in age, gender, or race across the three groups.

\section{Parent and Child Anxiety Ratings: ASD + Anxiety, ASD, and TD groups}

Mean and SD values of parent and child anxiety ratings across the three diagnostic groups are reported in Table 2. The mixed ANOVA revealed a significant main effect of group, $F(2,99)=54.18, P<0.001, \eta_{p}^{2}=0.52$, with highest anxiety ratings in the ASD + Anxiety group and lowest anxiety ratings in the TD group. There was also a significant interaction of group by rater, $F(2,99)=23.05, P<0.001, \eta_{p}^{2}=0.32$. Specifically, whereas parents rated anxiety higher than children in the ASD + Anxiety group, $t(33)=-3.81, P=0.001$, children rated anxiety higher than parents in the ASD and TD groups, $t(17)=3.13, P<0.01$ and $t(49)$ $=4.93, P<0.001$, respectively. Additionally, parent anxiety ratings were higher in the ASD + Anxiety group versus the ASD and TD groups, $t(50)=6.12, P<0.001$ and $t(37)=10.63$, $P<0.001$, respectively; however, child ratings were higher in the ASD + Anxiety group versus the TD group, $t(53)=4.62, P<0.001$, but comparable in the ASD + Anxiety and ASD groups $t(50)=0.13, P=0.90$. This interaction is depicted in Figure 1 .

There were moderate correlations between parent and child anxiety ratings in the ASD + Anxiety, $r=0.36, P<0.05$, and ASD groups, $r=0.53, P<0.05$, but not in the TD group, $r=$ $0.02, P>0.05$.

\section{Associations of Demographic and Clinical Characteristics with Parent-Child Discrepancy in Combined ASD Group}

Correlations between parent-child discrepancy score and child's age, gender, IQ, and ASD symptom severity are presented in Table 3. Parent-child discrepancy score was not associated with age, gender, or IQ. Of the indices of ASD symptom severity, parent-child discrepancy score was associated with ADI-R communication deficits, $r=0.33, P<0.05$, and ADI-R RRB, $r=0.29, P<0.05$. The association between parent-child discrepancy score and ADOS-2 SA approached significance, $r=0.29, P=0.06$. The scatterplots of significant associations are shown in Figures S1 and S2.

Based on the pattern of significant associations between parent-child discrepancy score and ADI-R communication deficits and ADI-R RRB, these two ADI-R variables were entered into a hierarchical linear regression predicting parent-child discrepancy score. To control for 
heterogeneity conferred by age, gender, and IQ, these variables were entered simultaneously in the first step of the model. ADI-R communication deficits and ADI-R RRB were entered simultaneously in the second step. The results of these analyses are reported in Table 4. After accounting for age, gender, and IQ, ADI-R communication deficits and ADI-R RRB, entered as a block, accounted for $14 \%$ of the variance in parent-child discrepancy, $P<0.05$. However, when included together in this step, neither ADI-R variable made a significant contribution. This pattern of results signifies that while neither communication deficits nor RRBs contributed uniquely to parent-child discrepancy, the overall level of ASD symptom severity evidenced by symptoms in these core areas contributed to discrepancy, and specifically the extent to which parent ratings exceeded child ratings.

\section{Discussion}

The present study is the first to compare parent and child anxiety ratings in children with ASD with and without comorbid anxiety disorders. The first aim of the study was to examine anxiety ratings in children with ASD with and without comorbid anxiety disorders relative to typically developing controls.

As expected, when combined over parent and child ratings, anxiety ratings were highest in the ASD + Anxiety group, lower in the ASD group, and lowest in the TD group. The tendency for children with ASD without comorbid anxiety disorders to have higher anxiety ratings than typically developing peers indicates that, even in the absence of clinically significant levels of anxiety, children with ASD experience notable anxiety symptomology. This is consistent with prior research documenting elevated anxiety in children with ASD and comparing children with ASD to non-ASD peers [Gillott, Furniss, \& Walter 2001; Greenaway \& Howlin 2010; Russell \& Sofronoff 2005]. This finding also suggests that anxiety symptoms exist along a continuum for children with ASD. In addition, as demonstrated in several previous studies [Blakeley-Smith et al. 2012; Ooi et al. 2016; White et al. 2012], parent and child anxiety ratings were moderately associated for children in both ASD groups. These findings are generally consistent with the low to moderate associations found between parent and child symptom ratings in the typically developing population [De Los Reyes et al. 2015].

Within the ASD + Anxiety group, both parents and children reported higher levels of anxiety relative to the TD group. The finding that children in the ASD + Anxiety group reported more anxiety than children in the TD group suggests that children with ASD and comorbid anxiety are aware of their anxiety to a degree and supports the notion that children with ASD are able to communicate about their emotional experiences. However, parent and child ratings in the ASD + Anxiety group also diverged significantly, with parent ratings exceeding child ratings. This finding is consistent with two studies that examined parent and child reports of anxiety in children with ASD and comorbid anxiety disorders [Storch et al. 2012; White et al. 2012]. Furthermore, parent ratings in the ASD + Anxiety group were in the clinically elevated range (MASC-2 Total Anxiety $T$-score 265 ) and higher than parent ratings in the ASD group. In contrast, child ratings in the ASD + Anxiety group were in the high average range (MASC-2 Total Anxiety $T$-score between 55 and 59) and comparable to child ratings in the ASD group, replicating earlier findings [Mazefsky et al. 2011]. These 
patterns of parent and child ratings may suggest a tendency for children with ASD and comorbid anxiety to have difficulty reporting their anxiety to its full extent and for their parents to be more aware of their anxiety, and particularly the extent to which it is clinically significant. Such findings are consistent with research suggesting that children with ASD underreport their anxiety symptoms [May et al. 2015; Mazefsky et al. 2011] and fail to report clinically significant levels of anxiety when present and determined by clinician consensus [Blakeley-Smith et al. 2012; Storch et al. 2012; White et al. 2012].

However, it is possible that differences between parent and child ratings in the ASD + Anxiety group may have been influenced by the methodology used to establish diagnostic status. Since comorbid anxiety disorders were diagnosed by semi-structured interviews with the parent and child, to the extent that parents may have reported more anxiety symptoms than children during the interviews, children who were assigned a comorbid anxiety diagnosis and placed in the ASD + Anxiety group may have been more likely to have higher parent ratings of anxiety. In such a case, obtained parent-child differences in anxiety ratings in the ASD + Anxiety group may reflect consistent patterns of responding across measurement instruments, with parents reporting higher levels of anxiety relative to their children in both the interview and rating scale formats. As such, the present finding of elevated parent- versus child-reported anxiety among children with ASD and comorbid anxiety should be further examined in studies supplemented by additional diagnostic methods.

The finding that children in the ASD + Anxiety and ASD groups reported subclinical levels of anxiety that were higher than those reported by their TD peers is consistent with research supporting the utility of child self-report among children with ASD [Blakeley-Smith et al. 2012; Ozsivadjian et al. 2014]. Moreover, this finding underscores the uniquely valuable role of child self-report in the assessment process. To the extent that self-report ratings reflect children's insights into their emotional experiences, endorsed self-report items can elucidate areas for more targeted assessment. For example, subsequent to collecting self-report ratings, a clinician-conducted interview focused on endorsed areas of concern could provide more information regarding the nature and extent of the child's anxiety while also scaffolding the child's general ability to report on his or her emotional experiences. Through such a process, the clinician may gain a more accurate and comprehensive picture of the child's anxiety symptoms, aided by the use of his or her self-report.

Consistent with earlier studies [Burrows et al. 2018; May et al. 2015], we found higher child versus parent ratings of anxiety in the TD group. This pattern may be explained by the presence of subclinical levels of anxiety in typically developing children and the tendency for parents to lack awareness of these symptoms [Ozsivadjian et al. 2014]. Similarly, we also found higher child versus parent ratings of anxiety in the ASD group. This finding is consistent with several studies examining children with ASD without established anxiety disorders [Hurtig et al. 2009; Magiati et al. 2014; Ooi et al. 2016]. Parents of children with ASD without anxiety disorders may, like parents of typically developing children, lack awareness of the subclinical, potentially less obvious, manifestations of their children's anxiety. 
The second aim of the study was to examine the association between ASD symptom severity and parent-child anxiety rating discrepancy. Consistent with our expectations, ASD symptom severity, marked by communication deficits and restricted and repetitive behaviors (RRBs), was positively associated with discrepancy in parent-child anxiety ratings. Age, gender, and IQ were not associated with parent-child anxiety rating discrepancy, indicating that these child characteristics did not contribute to differences in parent and child anxiety ratings in our sample. When tested in the regression model, ASD symptom severity predicted parent-child anxiety rating discrepancy, with greater symptom severity linked to the degree to which parent ratings exceeded child ratings. Notably, the combination of communication deficits and RRBs significantly predicted discrepancy score, although neither showed unique effects. This pattern suggests that overall level of ASD symptom severity, combined across the core domains of communication deficits and RRBs, contributes to the tendency for parents to rate their children's anxiety higher than their children. This finding is consistent with previous research demonstrating lower levels of parent-child anxiety rating agreement for children with more severe ASD symptomology [Kaat \& Lecavalier 2015].

Several factors may explain the positive association between ASD symptom severity and the pattern of higher parent versus child anxiety ratings found in the present study. First, deficits in social communication central to ASD, combined with limitations in emotional awareness, insight and understanding, may interfere with the ability to accurately use language to communicate about one's emotional experiences [Wood \& Gadow 2010]. Relatedly, deficits in communication may result in difficulty comprehending abstract, emotion-focused language characteristic of items on self-report rating scales [Blakeley-Smith et al. 2012]. Together, these difficulties with both expressive and receptive components of language may hinder the ability to endorse experiences of anxiety via self-report, leading to reduced child anxiety ratings. In addition, many of the RRBs commonly engaged in by children with ASD (e.g., ritualized patterns of behaviors, insistence on sameness) may be interpreted by parents as manifestations of anxiety [Storch et al. 2012], leading to heightened parent ratings of anxiety. In these ways, by contributing to reduced child ratings and/or heightened parent ratings, ASD symptom severity may augment parent-child anxiety rating discrepancy.

The association between ASD symptom severity and parent-child anxiety rating discrepancy has relevant implications for clinical practice. Notably, this finding underscores the importance of considering children's levels of ASD severity when interpreting parent and child anxiety ratings and using them to inform diagnostic evaluation. To the extent that core ASD symptoms, including social communication deficits and RRBs, impact parent and child anxiety ratings in ways that attenuate the accuracy of these ratings, additional assessment methods aimed at facilitating accurate reporting are advised. For children, such methods may include unstructured interviews intended to probe areas of endorsed concern, as well as ratings scales with modified language and clarifying elements (e.g., objective examples, rating scale anchors) aimed at addressing potential comprehension difficulties [BlakeleySmith et al. 2012]. Furthermore, to increase the utility of parent reports of child anxiety, assessment measures that more precisely index symptoms of anxiety separate from core features of ASD are needed. Efforts in this area are already underway [Scahill et al. 2019] and provide a promising avenue of research. 
The presence of parent-child anxiety rating discrepancies may also reflect other clinically meaningfully patterns and thus signal areas for continued assessment. Specifically, to the degree that parent-child disagreement regarding a child's behavioral and emotional problems reflects broader aspects of the parent-child relationship, this disagreement may be associated with parent-child interactions that contribute to the child's emotional difficulties [De Los Reyes \& Kazdin 2006]. For example, for a certain parent-child dyad for whom anxiety-rating disagreement is associated with limited parent-child conversations regarding emotions, the lack of such conversations may preclude parent-mediated scaffolding of emotion regulation skills needed for the child's effective management of anxiety. By considering the ways in which parent-child anxiety discrepancies may be related to other aspects of child and family functioning, and by collecting additional information to better understand these patterns, clinicians may be able to use discrepancies to gain a more thorough conceptualization of a child's anxiety and overall functioning.

Several limitations of the present study warrant mention. First, the cross-sectional design of the study precludes inferences regarding the direction of the association between ASD symptom severity and parent-child anxiety rating discrepancy. Studies employing longitudinal designs are needed to examine the link between ASD symptomology and anxiety ratings over time and test causal associations. Second, the sample size of the combined ASD group in the current study was small, limiting the power to detect effects. Studies with larger samples are needed to further explore the influences of communication deficits and RRBs on parent-child anxiety rating discrepancy. Studies with larger samples may also be useful for exploring differences in parent-child rating discrepancies specific to particular anxiety disorders. Third, in this study, children with ASD and their families were seeking treatments, including treatments for anxiety co-occurring with autism. As such, our findings may not be generalizable to non-referred populations. This limitation can be addressed in epidemiological studies with larger samples. Fourth, we cannot be certain that the between-group differences in parent and child anxiety ratings were not influenced by aspects of the characterization process and differences in relative weight placed on parent versus child report during the diagnostic interviews. This limitation of psychiatric diagnoses that are based on collecting information from children and their parents can be addressed by developing more objective measures of anxiety. Lastly, the present study sample included mostly boys. Future studies should focus on exploring patterns of parent-child anxiety rating discrepancy in girls with ASD.

The present study is the first to compare parent and child anxiety ratings in children with ASD with and without comorbid anxiety disorders. Findings suggest that while the overall scores derived from child anxiety ratings should be interpreted with caution, child ratings reflect important information regarding the anxiety symptoms of which children are aware and are likely to provide a valuable tool for the comprehensive assessment of anxiety. Additionally, both parent and child anxiety ratings should be considered in light of children's ASD symptom severity and used to inform continued assessment aimed at further elucidating children's patterns of anxiety. Future research should focus on the development of assessment tools aimed at increasing the utility of parent-and self-report of anxiety in children with ASD. 


\section{Supplementary Material}

Refer to Web version on PubMed Central for supplementary material.

\section{Acknowledgments}

This work was supported by NIMH Grant R01MH101514 (D.G.S.) and NICHD Grant R01HD083881 (D.G.S.). C.B.K. and K.I. are Fellows of the Translational Developmental Neuroscience Training Program (T32 MH18268) directed by Dr. Michael Crowley.

\section{References}

Achenbach TM (2006). As others see us - Clinical and research implications of cross-informant correlations for psychopathology. Current Directions in Psychological Science, 15, 94-98. 10.1111/ j.0963-7214.2006.00414.x

Bal E, Harden E, Lamb D, Van Hecke AV, Denver JW, \& Porges SW (2010). Emotion recognition in children with autism spectrum disorders: Relations to eye gaze and autonomic state. Journal of Autism and Developmental Disorders, 40, 358-370. 10.1007/s10803-009-0884-3 [PubMed: 19885725]

Bitsika V, \& Sharpley CF (2015). A comparison of self-vs parent reports of generalised anxiety disorder symptomatology across six age groups for boys with an ASD. Journal of Developmental and Physical Disabilities, 27, 249-261. 10.1007/s10882-014-9413-1

Blakeley-Smith A, Reaven J, Ridge K, \& Hepburn S (2012). Parent-child agreement of anxiety symptoms in youth with autism spectrum disorders. Research in Autism Spectrum Disorders, 6, 707-716. 10.1016/j.rasd.2011.07.020

Bujang MA, \& Baharum N (2017). Guidelines of the minimum sample size requirements for Cohen's Kappa. Epidemiology, Biostatistics and Public Health, 14, e12267-12261-e12267-12210. $10.2427 / 12267$

Burrows CA, Usher LV, Becker-Haimes EM, McMahon CM, Mundy PC, Jensen-Doss A, \& Henderson HA (2018). Profiles and correlates of parent-child agreement on social anxiety symptoms in youth with autism spectrum disorder. Journal of Autism and Developmental Disorders, 48, 2023-2037. 10.1007/s10803-018-3461-9 [PubMed: 29332179]

De Los Reyes A, Augenstein TM, Wang M, Thomas SA, Drabick DAG, Burgers DE, \& Rabinowitz J (2015). The validity of the multi-informant approach to assessing child and adolescent mental health. Psychological Bulletin, 141, 858-900. 10.1037/a0038498 [PubMed: 25915035]

De Los Reyes A, \& Kazdin AE (2004). Measuring informant discrepancies in clinical child research. Psychological Assessment, 16, 330-334. 10.1037/1040-3590.16.3.330 [PubMed: 15456389]

De Los Reyes A, \& Kazdin AE (2005). Informant discrepancies in the assessment of childhood psychopathology: A critical review, theoretical framework, and recommendations for further study. Psychological Bulletin, 131, 483-509. 10.1037/0033-2909.131.4.483 [PubMed: 16060799]

De Los Reyes A, \& Kazdin AE (2006). Informant discrepancies in assessing child dysfunction relate to dysfunction within mother-child interactions. Journal of Child and Family Studies, 15, 643-661. 10.1007/s10826-006-9031-3 [PubMed: 21243074]

Duvekot J, van der Ende J, Verhulst FC, \& Greaves-Lord K (2018). Examining bidirectional effects between the autism spectrum disorder (ASD) core symptom domains and anxiety in children with ASD. Journal of Child Psychology and Psychiatry, 59, 277-284. 10.1111/jcpp.12829 [PubMed: 29076153]

Elliott CD (2007). The differential abilities scales, second edition (DAS-II). San Antonio, TX: Pearson Education, Inc.

Farrugia S, \& Hudson J (2006). Anxiety in adolescents with Asperger syndrome: Negative thoughts, behavioral problems, and life interference. Focus on Autism and Other Developmental Disabilities, $21,25-35$. 
Foley DL, Rutter M, Angold A, Pickles A, Maes HM, Silberg JL, \& Eaves LJ (2005). Making sense of informant disagreement for overanxious disorder. Journal of Anxiety Disorders, 19, 193-210. 10.1016/j.janxdis.2004.01.006 [PubMed: 15533704]

Gillott A, Furniss F, \& Walter A (2001). Anxiety in high-functioning children with autism. Autism, 5, 277-286. 10.1177/1362361301005003005 [PubMed: 11708587]

Greenaway R, \& Howlin P (2010). Dysfunctional attitudes and perfectionism and their relationship to anxious and depressive symptoms in boys with autism spectrum disorders. Journal of Autism and Developmental Disorders, 40, 1179-1187. 10.1007/s10803-010-0977-z [PubMed: 20182783]

Hallett V, Lecavalier L, Sukhodolsky DG, Cipriano N, Aman MG, McCracken JT, ... Scahill L (2013). Exploring the manifestations of anxiety in children with autism spectrum disorders. Journal of Autism and Developmental Disorders, 43, 2341-2352. 10.1007/s10803-013-1775-1 [PubMed: 23400347]

Hunsley J, \& Mash EJ (2007). Evidence-based assessment. Annual Review of Clinical Psychology, 3, 29-51. 10.1146/annurev.clinpsy.3.022806.091419

Hurtig T, Kuusikko S, Mattila ML, Haapsamo H, Ebeling H, Jussila K, ... Moilanen I (2009). Multiinformant reports of psychiatric symptoms among high-functioning adolescents with Asperger syndrome or autism. Autism, 13, 583-598. 10.1177/1362361309335719 [PubMed: 19933765]

Kaat AJ, \& Lecavalier L (2015). Reliability and validity of parent- and child-rated anxiety measures in autism spectrum disorder. Journal of Autism and Developmental Disorders, 45, 3219-3231. 10.1007/s10803-015-2481-y [PubMed: 26036649]

Kaufman J, Birmaher B, Axelson D, Perepletchikova F, Brent D, \& Ryan N (2016). Schedule for affective disorders and schizophrenia for school aged children: Present and lifetime version for DSM-5 (K-SADS-PL). Retrieved from http://www.pediatricbipolar.pitt.edu/resources/instruments.

Landis JR, \& Koch GG (1977). The measurement of observer agreement for categorical data. Biometrics, 33, 159-174. [PubMed: 843571]

Le Couteur A, Lord C, \& Rutter M (2003). The autism diagnostic interview-revised (ADI-R). Los Angeles, CA: Western Psychological Services.

Lecavalier L, McCracken CE, Aman MG, McDougle CJ, McCracken JT, Tierney E, ... Scahill L (2019). An exploration of concomitant psychiatric disorders in children with autism spectrum disorder. Comprehensive Psychiatry, 88, 57-64. 10.1016/j.comppsych.2018.10.012 [PubMed: 30504071]

Leckman JF, Sholomskas D, Thompson WD, Belanger A, \& Weissman MM (1982). Best estimate of lifetime psychiatric diagnosis: A methodological study. Archives Of General Psychiatry, 39, 879883. [PubMed: 7103676]

Lopata C, Toomey JA, Fox JD, Volker MA, Chow SY, Thomeer ML, ... Smerbeck AM (2010). Anxiety and depression in children with HFASDs: symptom levels and source differences. Journal of Abnormal Child Psychology, 38, 765-776. 10.1007/s10802-010-9406-1 [PubMed: 20354899]

Lord C, Rutter M, DiLavore PC, Risi S, Gotham K, \& Bishop SL (2012). Autism diagnostic observation schedule: ADOS-2. Los Angeles, CA: Western Psychological Services.

Losh M, \& Capps L (2006). Understanding of emotional experience in autism: Insights from the personal accounts of high-functioning children with autism. Developmental Psychology, 42, 809818. 10.1037/0012-1649.42.5.809 [PubMed: 16953688]

Magiati I, Chan JY, Tan WLJ, \& Poon KK (2014). Do non-referred young people with Autism Spectrum Disorders and their caregivers agree when reporting anxiety symptoms? A preliminary investigation using the Spence Children's Anxiety Scale. Research in Autism Spectrum Disorders, 8, 546-558. 10.1016/j.rasd.2014.01.015

March JS (2012). Multidimensional anxiety scale for children 2nd edition (MASC 2). Toronto, CA: MultiHealth Systems.

May T, Cornish K, \& Rinehart NJ (2015). Parent-child agreement using the spence children's anxiety scale and a thermometer in children with autism spectrum disorder. Autism Research and Treatment, 2015, 315495 10.1155/2015/315495 [PubMed: 25922765]

Mazefsky CA, Kao J, \& Oswald DP (2011). Preliminary evidence suggesting caution in the use of psychiatric self-report measures with adolescents with high-functioning autism spectrum disorders. 
Research in Autism Spectrum Disorders, 5, 164-174. 10.1016/j.rasd.2010.03.006 [PubMed: 24013401]

Miller LD, Martinez YJ, Shumka E, \& Baker H (2014). Multiple informant agreement of child, parent, and teacher ratings of child anxiety within community samples. The Canadian Journal of Psychiatry, 59, 34-39. 10.1177/070674371405900107 [PubMed: 24444322]

Ooi YP, Weng SJ, Magiati I, Ang RP, Goh TJ, Fung DS, \& Sung M (2016). Factors influencing agreement between parent and child reports of anxiety symptoms among children with highfunctioning autism spectrum disorders. Journal of Developmental and Physical Disabilities, 28, 407-424. 10.1007/s10882-016-9481-5

Ozsivadjian A, Hibberd C, \& Hollocks MJ (2014). Brief report: The use of self-report measures in young people with autism spectrum disorder to access symptoms of anxiety, depression and negative thoughts. Journal of Autism and Developmental Disorders, 44, 969-974. 10.1007/ s10803-013-1937-1 [PubMed: 24014195]

Russell E, \& Sofronoff K (2005). Anxiety and social worries in children with Asperger syndrome. Australian and New Zealand. Journal of Psychiatry, 39, 633-638. 10.1111/ j.1440-1614.2005.01637.x

Scahill L, Lecavalier L, Schultz RT, Evans AN, Maddox B, Pritchett J, ... Edwards MC (2019). Development of the parent-rated anxiety scale for youth with autism spectrum disorder. Journal of the American Academy of Child \& Adolescent Psychiatry, 58(9), 887-896.e2.

Silverman W, \& Albano AM (1996). Anxiety disorders interview schedule for DSM-IV, child version. New York, NY: Oxford University Press.

Silverman WK, \& Ollendick TH (2005). Evidence-based assessment of anxiety and its disorders in children and adolescents. Journal of Clinical Child \& Adolescent Psychology, 34, 380-411. 10.1207/s15374424jccp3403_2

Storch EA, Ehrenreich May J, Wood JJ, Jones AM, De Nadai AS, Lewin AB, ... Murphy TK (2012). Multiple informant agreement on the anxiety disorders interview schedule in youth with autism spectrum disorders. Journal of Child and Adolescent Psychopharmacology, 22, 292-299. 10.1089/ cap.2011.0114 [PubMed: 22856332]

Sukhodolsky DG, Bloch MH, Panza KE, \& Reichow B (2013). Cognitive-behavioral therapy for anxiety in children with high-functioning autism: a meta-analysis. Pediatrics, 132, e1341-e1350. 10.1542/peds.2013-1193 [PubMed: 24167175]

Sukhodolsky DG, Scahill L, Gadow KD, Arnold LE, Aman MG, McDougle CJ, ... Vitiello B (2008). Parent-rated anxiety symptoms in children with pervasive developmental disorders: frequency and association with core autism symptoms and cognitive functioning. Journal of Abnormal Child Psychology, 36, 117-128. 10.1007/s10802-007-9165-9 [PubMed: 17674186]

Wechsler D (1999). Wechsler abbreviated scale of intelligence. San Antonio, TX: The Psychological Corporation.

White SW, Schry AR, \& Maddox BB (2012). Brief report: The assessment of anxiety in highfunctioning adolescents with autism spectrum disorder. Journal of Autism and Developmental Disorders, 42, 1138-1145. 10.1007/s 10803-011-1353-3 [PubMed: 21874396]

Wood JJ, \& Gadow KD (2010). Exploring the nature and function of anxiety in youth with autism spectrum disorders. Clinical Psychology-Science and Practice, 17, 281-292. 10.1111/ j.1468-2850.2010.01220.x 


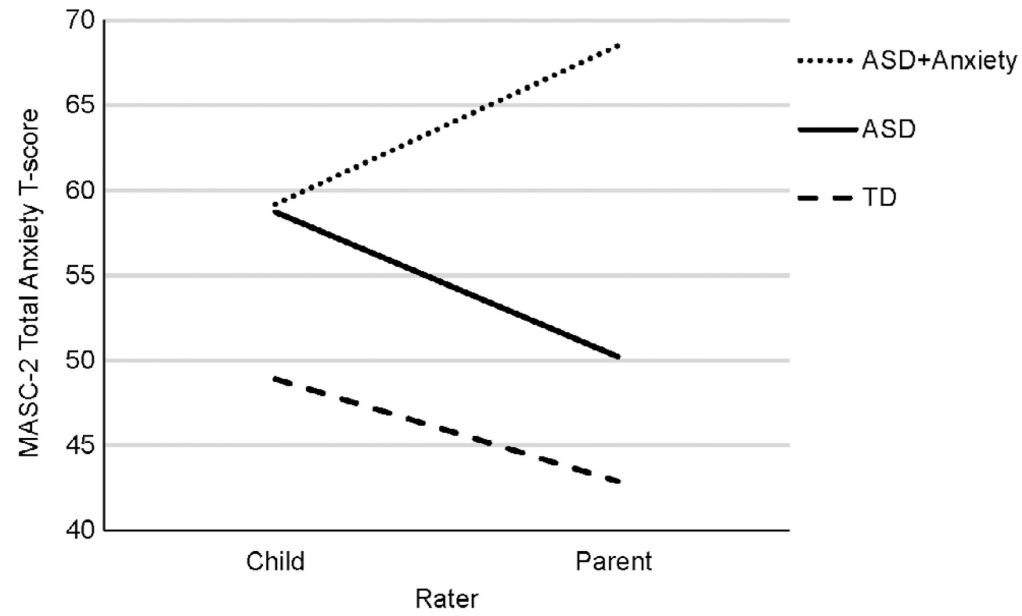

Figure 1.

Results of a mixed-design ANOVA examining differences in anxiety ratings across raters (parent, child) and diagnostic groups (children with ASD and comorbid anxiety, ASD +Anxiety; children with ASD without comorbid anxiety, ASD; typically developing healthy controls, TD). Anxiety ratings correspond to MASC-2 Total Anxiety T-scores. 


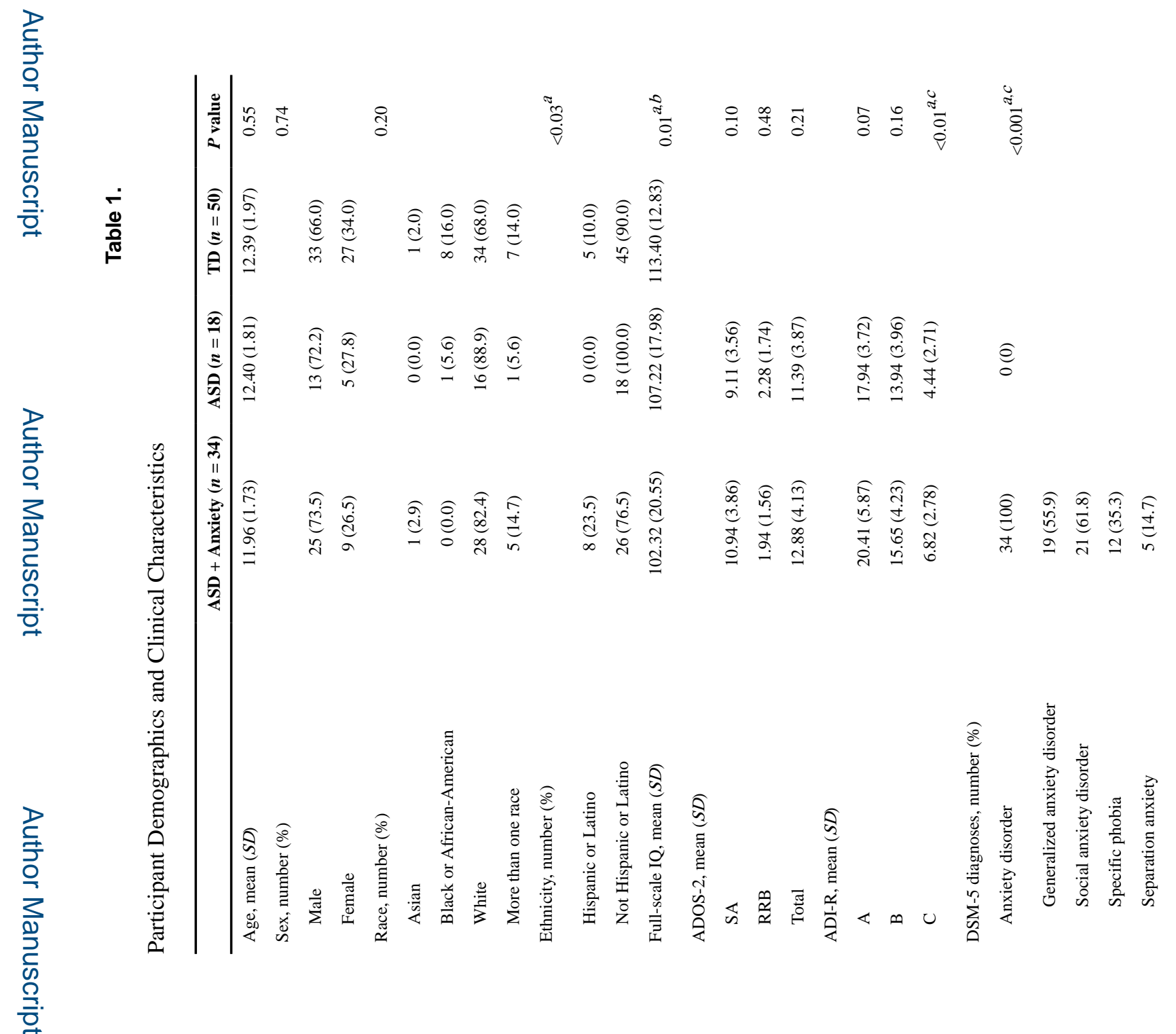
Autism Res. Author manuscript; available in PMC 2020 March 09. 


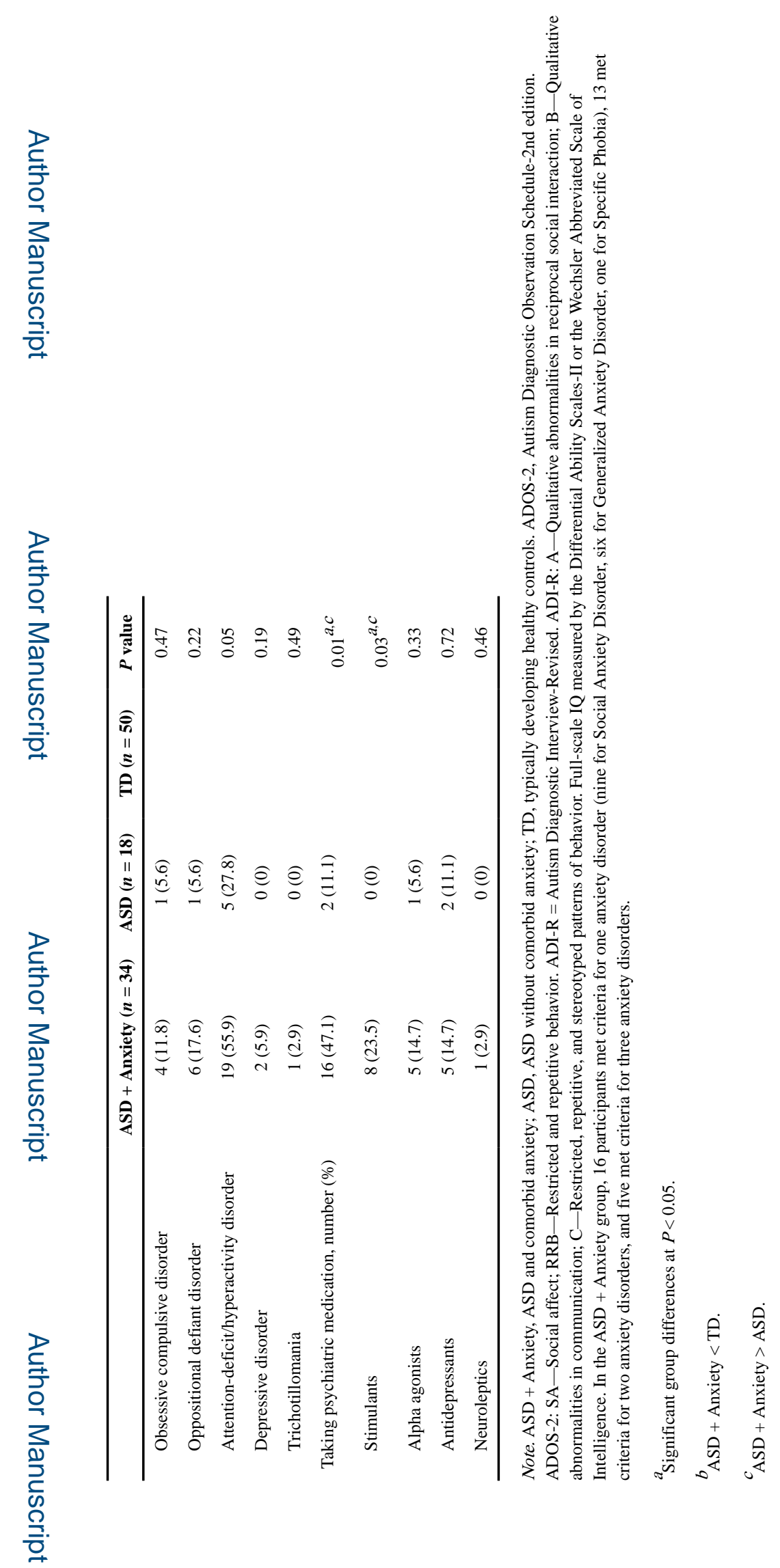


Table 2.

Between-Group Differences in Parent and Child Anxiety Ratings

\begin{tabular}{lccc}
\hline & ASD + Anxiety $(\boldsymbol{n}=\mathbf{3 4})$ & $\operatorname{ASD}(\boldsymbol{n}=\mathbf{1 8})$ & $\mathbf{T D}(\boldsymbol{n}=\mathbf{5 0})$ \\
\hline MASC-2 total anxiety $T$-score, mean $(S D)$ & & & \\
$\quad$ Parent report & $68.50(13.63)$ & $50.22(7.89)$ & $42.88(4.12)$ \\
$\quad$ Child self-report & $59.18(11.31)$ & $58.72(13.60)$ & $48.90(7.67)$ \\
\hline
\end{tabular}

Note. ASD + Anxiety = ASD and comorbid anxiety; ASD = ASD without comorbid anxiety; TD = typically developing healthy controls. 
Table 3.

Correlations between Parent-Child Anxiety Discrepancy Score and Age, Gender, IQ, and ASD Severity in the ASD Combined Group (ASD + Anxiety plus ASD, $n=52$ )

\begin{tabular}{lc}
\hline & Parent-child discrepancy score \\
\hline Gender & 0.11 \\
Age & 0.04 \\
Full-scale IQ & -0.06 \\
ADOS-2 SA & 0.26 \\
ADOS-2 RRB & -0.05 \\
ADOS-2 Total & 0.23 \\
ADI-R A & 0.22 \\
ADI-R B & $\mathbf{0 . 3 3}^{*}$ \\
ADI-R C & $\mathbf{0 . 2 9}^{*}$ \\
\hline
\end{tabular}

Note. ADOS-2, Autism Diagnostic Observation Schedule-2nd edition. ADOS-2: SA—Social affect; RRB—restricted and repetitive behavior. ADI$\mathrm{R}=$ Autism Diagnostic Interview-Revised. ADI-R: A-Qualitative abnormalities in reciprocal social interaction; B-Qualitative abnormalities in communication; C-Restricted, repetitive, and stereotyped patterns of behavior. Parent-child discrepancy score $=$ standardized parent MASC-2 Total Anxiety $T$-score - standardized child MASC-2 Total Anxiety $T$-score.

${ }^{*} P<0.05$. 
Table 4.

Hierarchical Regression Analysis of Parent-Child Anxiety Discrepancy Score as a Function of ASD Severity in the ASD Combined Group (ASD + Anxiety plus ASD, $n=52$ )

\begin{tabular}{lccc}
\hline Variable & $\boldsymbol{R}^{\mathbf{2}}$ & $\boldsymbol{\beta}$ & $\boldsymbol{\Delta} \boldsymbol{F}$ \\
\hline Step 1 & 0.01 & & 0.23 \\
Age & & 0.05 & \\
Gender & & 0.10 & \\
IQ & & -0.03 & \\
Step 2 & 0.16 & & $3.90^{*}$ \\
ADI-R B & & 0.29 & \\
ADI-R C & & 0.21 & \\
\hline
\end{tabular}

Note. ADI-R, Autism Diagnostic Interview-Revised. ADI-R subscales: B-Qualitative abnormalities in communication; C-Restricted, repetitive, and stereotyped patterns of behavior. Parent-child discrepancy score $=$ standardized parent MASC-2 Total Anxiety $T$-score - standardized child MASC-2 Total Anxiety $T$-score.

* $P<0.05$. 